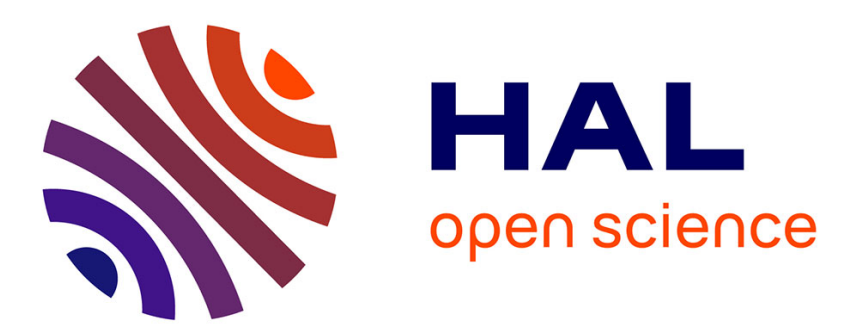

\title{
Association of power control and data rate selection in WCDMA
}

Loutfi Nuaymi

\section{To cite this version:}

Loutfi Nuaymi. Association of power control and data rate selection in WCDMA. PIMRC 2003 14th IEEE international symposium on personal indoor and mobile radio communications, Sep 2003, Beijing, China. 10.1109/PIMRC.2003.1264282 . hal-02128339

\section{HAL Id: hal-02128339 \\ https://hal.science/hal-02128339}

Submitted on 14 May 2019

HAL is a multi-disciplinary open access archive for the deposit and dissemination of scientific research documents, whether they are published or not. The documents may come from teaching and research institutions in France or abroad, or from public or private research centers.
L'archive ouverte pluridisciplinaire HAL, est destinée au dépôt et à la diffusion de documents scientifiques de niveau recherche, publiés ou non, émanant des établissements d'enseignement et de recherche français ou étrangers, des laboratoires publics ou privés. 


\title{
Association of Power Control and Data Rate Selection in WCDMA
}

\author{
Loutfi Nuaymi \\ ENST Bretagne, 2, Rue de la Chataigneraie, B.P. 78, Cesson Sévigné 35512 Cedex, FRANCE \\ e-mail: loutfi.nuaymi@enst-bretagne.fr
}

\begin{abstract}
In this paper, we study the interaction between power control and data rate selection in a wireless CDMA network. Specifically, third-generation cellular WCDMA system is considered. The criterion is the average of the sum of data rates in a variable rates services environment. Simulation results show that the association (or the integration) of power control and data rate selection allows a given network to have better achievable performance. Interesting elements of the proposed algorithm are highlighted and useful comments given.
\end{abstract}

\section{Introduction}

One of the major innovations of third-generation cellular networks is the presence of different communication services including different data rates, which can have relatively high values. Data rates can reach 2 or more $\mathrm{Mb} / \mathrm{s}$. The multiple access system selected for third-generation cellular systems is Code-Division Multiple Access (CDMA). For wireless CDMA systems, capacity limitations are mainly due to interference ([1]). Radio resource management allows lower interference and thus higher capacity. In this paper, we consider the application of an algorithm combining power control and data rate selection for WCDMA.

For WCDMA ([2]), the European and Japanese common proposal for third-generation IMT-2000, power control is defined in [3] for Frequency-Division Duplex (FDD) system and [4] for Time-Division Duplex (TDD) system.

In a multimedia environment such as in WCDMA, different services with different constraints are transmitted simultaneously. In this paper, we try to optimise resource allocation (selection of transmitted power and rate) in a wireless network where each communication has different possible data rates, including zero (no-transmission). This is, for example, the case of Internet communications. The criterion of optimisation is the instantaneous sum of data rates. Evidently this is a restrictive hypothesis since in real networks some services will have a mandatory minimal data rate in addition to transmission delay constraints; this is, for example, the case of voice transmission. Yet, the computation of the maxinial sum of data rates can be useful for a general wireless multimedia CDMA system.

Considering a given user and rate distribution, the existence of a transmitted power vector such that all communications verify their quality constraint has been discussed in [5]. Integrated power control and rate selection for the uplink of a cellular CDMA network with two classes of users, typically voice and data users, is studied in [6]. A transmission scheduling scheme for delay-tolerant users allowing relatively higher throughput and lower intercell interference, while respecting different quality requirements, is proposed. The same topic for downlink non real-time services is considered in [7]. Scheduling is such that one user transmits at a time in each cell. A distributed power control algorithm allows intercell interference management. Other references about the association of power control and data rate can be found in [7].

In this paper, we study uplink variable data rate services. A simple approach is used in order to show interesting results of capacity increase when power control and data rate are associated. We start by reviewing some previously-proposed studies on that topic. The system model considered is described and some details about power control and data rates in WCDMA given. Then we propose some interesting results concerning the total throughput of a CDMA cell. A discussion of the results and conclusions are given in the last section. Although the implementation topic is not directly addressed in our paper, the results and the comparisons obtained may be used for performance evaluation.

\section{System model}

The uplink (mobile-to-station) of a CDMA $3 \mathrm{G}$ system is considered. In an FDD-CDMA system, an uplink physical channel is defined by its code and its frequency. The methods proposed in this paper could be applied to downlink and/or to TDD systems after some slight adaptations. Power control is only applied at Layer 1 (inner closed-loop PC). Circuitswitched systems are considered. Packet transmission for packet-switched services is assumed to be taken into account at layer 3 or higher layers.

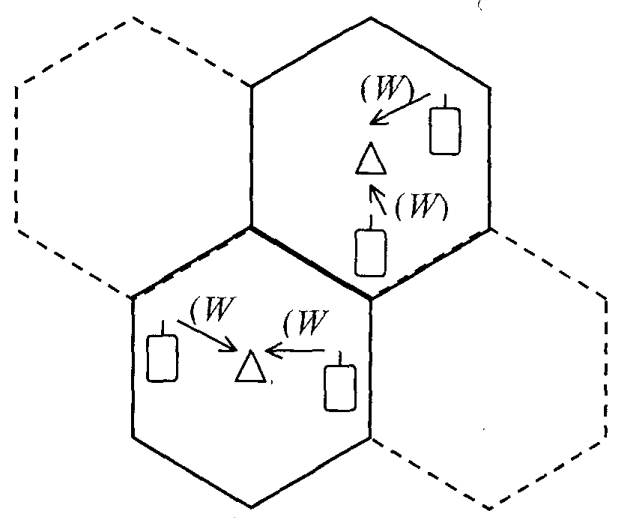

Fig. I: Reverse link of a CDMA cellular network. All mobiles use the same Frequency Bandwidth $W$. 
A fairly simple simulation model is used. This allows an illustration of the results of this work. A maximal transmitted power limitation is considered. The simulation model consists of $M$ mobiles that transmit information sharing a common radio channel (uplink of a cellular CDMA system). Each mobile should communicate with one of the $B$ base stations (BS). Interference between the reverse links of a CDMA network is illustrated in Fig. 1.

The algorithms considered are applied for a fixed set of mobiles, their application being supposed fast enough to consider the mobile set and geographical positions all fixed (snapshot). The speed of convergence of the iterative algorithms is verified in the following.

The index of the base to which mobile $m$ is assigned is $a_{n l}$. A mobile is connected to the best-received power level base station (sometimes called the best-server), which is not always the closest one due to the fadings. Mobile $m$ transmitted power is $p_{m}$ and the link gain from mobile $m$ to base $b$ is noted $g_{b m}$, so matrix $G=\left(g_{h m}\right)$ is of size ( $\left.B \times M\right)$. Mobile $m$ received signalto-interference ratio (SIR) on base $b$, noted $\gamma_{m, b}$, is then given by:

$$
\gamma_{m, h}=\frac{g^{\prime m} p_{m}}{\sum_{j=1 ; j \neq m}^{M} g_{b j} p_{j}+n h}
$$

where $n_{h}$ is the receiver noise, also called thermal noise, at base $b$. The received SIR of mobile $m$ on its assigned BS is noted $\gamma_{m}$, so we can write: $\gamma_{m}=\gamma_{m, a_{m}}$. A mobile is in outage state when its SIR falls below its SIR target.

In a CDMA system, the received BER is a direct function of the energy-per-bit over total noise spectral density ratio, noted $E_{b} / N_{0}$. Thus, $E_{h} / N_{0}$ is considered as a good quality indicator. It is related to the received SIR, noted $\gamma$, by the following formula:

$$
\gamma=\frac{E_{b}}{N_{0}} \frac{R}{W}
$$

where $R$ is the data (or information) bit rate and $W$ the total frequency bandwidth ( $5 \mathrm{MHz}$ for WCDMA). The value $E_{b} / N_{0}$ is sometimes called the despread SIR. The spreading factor (SF), or $W / R$, is then an important parameter for the decision of the quality threshold.

For a data rate $r^{k}$ and for a required threshold for $E_{h} / N_{0}$, noted $\left(\frac{E_{b}}{N_{0}}\right)_{T}$, the threshold for the received SIR, noted $\omega_{k}$, is given by:

$$
\omega_{k}=\left(\frac{E_{b}}{N_{0}}\right)_{T}^{k} \frac{r_{k}}{W}
$$

Communications have different possible data rates and $E_{b} / N_{0}$ targets. Thus, different SIR targets are considered. In this work, we consider that each data communication has $D$ possible data rates :

$$
r^{1}<r^{2}<\ldots<r^{D}
$$

where $r^{l}$ is equal to zero (corresponding to a no-transmission state).

Paging or synchronization channels are not included in the simulation. Performance of the algorithm is evaluated by the average sum of transmitted rates. More details about the simulation are given below in the following sections.

\section{Power control and data rate selection in WCDMA system}

Power control in WCDMA is a closed-loop power control (PC) which is a combination of the outer and inner closed loop controls. The inner (also called fast) closed loop PC adjusts the transmitted power in order to keep the received SIR equal to a given target. This SIR target is fixed according to the received Bit Error Rate (BER) by the higher layer outer-loop PC in order to achieve a given BLock Error Rate (BLER) target. Ensuring that the lowest possible SIR target is used results in greater network capacity. The BER target is a function of the communication service. In this paper, we use fixed values for SIR thresholds (see below). The results should still hold for varying values of SIR thresholds (e.g., by the outer-loop).

The inner closed-loop PC measures the received quality, represented by received SIR and then sends commands to the transmitter (i.e., the mobile in the case of the uplink) for the power update. The receiver estimates the received power of the connection to be power controlled. Simultaneously, the receiver estimates the received interference. The obtained SIR estimate, noted $\mathrm{SIR}_{\text {est }}$, is then used by the receiver to generate PC commands.

The frame duration in WCDMA System is $10 \mathrm{~ms}$ and contains 15 time slots. The transmitted power is fixed for a given time slot. Each slot then has one PC information bit, which gives a PC update rate of $1500 \mathrm{~b} / \mathrm{s}$. Each PC command increases or decreases the transmitted power level by a fixed value of around $1 \mathrm{~dB}$, according to Algorithm 1 or 2 of [8]. In this work, the power update step size is always equal to $1 \mathrm{~dB}$. We studied Adaptive-Step Power Control for WCDMA in [9].

Fast PC is made by the OSI model (PHYsical) Layer 1. Outer-loop PC is realized by Layer 3 RRC (Radio Resource Control). The outer loop PC update frequency is $10-100 \mathrm{~Hz}$ ([2]). RRC can also make the data rate selection.

\section{Dato rates and SIR thresholds}

In WCDMA System, different data rates can be used ([8]). In our simulation, we considered the uplink data rates of the DPCH (Dedicated Physical Channel), rewritten in Table 1. The threshold of $E_{b} / N_{0}$ corresponding to a given BER is theoretically independent of the data rate. In WCDMA system, I the energy of the pilot bits is more important for high data rates than for smaller rates. This leads to easier demodulation, and then smaller $E_{b} / N_{0}$ thresholds, for higher data rates than for smaller data rates. 
For a given BER, the value of the $E_{h} / N_{0}$ threshold is a function of many factors: modulation, coding, average mobile speed, radio channel details (multipath, etc), receiver type, antenna type and diversity and other operating environment parameters. In our simulation we used the values of Table 1 for $E_{N} / N_{0}$ thresholds. According to what is said above for WCDMA, these values decrease when the data rates increase. Formula (3) is then used for the computation of the SIR threshold for the different data rates (and thus the different spreading factors). Results of this paper still hold for different values of $E_{h} / N_{0}$ thresholds, and even for time-varying $E_{h} / N_{0}$ thresholds, e.g., as a result of outer-loop PC.

The association of data rate selection and power control has many advantages: one of these is the possibility of choosing the highest data rate that has an achievable SIR threshold. This choice can be updated according to the network parameters (and, specifically, saturation) for communication services where it is possible.

\begin{tabular}{|c|c|c|c|}
\hline $\begin{array}{c}\text { Data rate } \\
\text { (Kbits/s) }\end{array}$ & $\begin{array}{c}\mathbf{E}_{\mathrm{b}} / \mathbf{N}_{0} \\
(\mathbf{d B})\end{array}$ & $\begin{array}{c}\text { SIR } \\
\text { (real valueshold }\end{array}$ & $\begin{array}{c}\mathbf{S I R}_{\text {Threshold }} \\
(\mathbf{d B})\end{array}$ \\
\hline 15 & 7 & 0.015 & -18.2 \\
\hline 30 & 6 & .0 .023 & -16.4 \\
\hline 60 & 6 & 0.047 & -13.3 \\
\hline 120 & 5 & 0.075 & -11.2 \\
\hline 240 & 5 & 0.15 & -8.2 \\
\hline 480 & 4 & 0.24 & -6.2 \\
\hline 960 & 3 & 0.38 & -4.2 \\
\hline
\end{tabular}

Table 1. WCDMA data rates. The thresholds for $E_{b} / N_{0}$ used in our simulation and corresponding signal-to-interference ratios are also given.

In the remainder of this paper, we consider that all the communications can at any instant have one of the data rates of Table 1. Rate 0 (i.e., no transmission) is also possible. This could be the case for Internet-style communication. Obviously, in real networks, some services will have a fixed rate or a mandatory minimal data rate in addition to transmission delay constraint; this is, for example, the case of voice transmission. However, the computation of the maximal sum of data rates, with the hypothesis we consider, can be useful for a general wireless multimedia CDMA system.

\section{Power control and rate selection}

Power control is a part of the radio resource control in a CDMA system. Association of the power control and the base station selection is studied in [10].

The association of the processes of power control and data rate selection can be made by choosing simultaneously and according to a given criterion the transmitted power and the data rate for each transmitter (mobile or base station). These choices can be made by a centralized or a distributed algorithm.

The Selective Power Control (SPC) Algorithm proposed in [5] is a generalization of the power control algorithm known as Distributed Power Control (DPC) of [11] and [12]. The SPC tries to maximize the sum of data rates with minimal energy consumption. It is an iterative distributed algorithm.

This iterative algorithm is applied for a fixed set of mobiles, its application being assumed fast enough to consider the mobile set and geographical positions all fixed (snapshot). The transmitted power of mobile $i$ at instant $v$ is noted $p_{i}^{(v)}$, where the time index is $v$. Its received SIR is noted $\gamma_{i}(v)$. The maximum (resp. minimum) transmitted power is $P_{M a x}$ (resp. $\left.P_{\text {Min }}\right)$. Each transmitter data rate and transmitted power is computed, iteratively, as follows. Starting from an arbitrary initial transmitted power vector $\boldsymbol{P}^{(0)}$, the next iteration transmitted power is determined by:

$$
p_{i}(v+1)=\operatorname{Max}_{k}\left\{\frac{p_{i}(v) \omega_{k}}{\gamma_{i}(v)} \cdot \chi\left(\frac{p_{i}(v) \omega_{k}}{\gamma_{i}(v)} \leq P_{M a x}\right)\right\},
$$

where $(E)$ is the indicator function of the event $E$. The data rate of mobile $i$, at convergence (or at any instant $v$ ) is noted $r_{\text {index_i(v) }}$, where index_i(v) is given by:

$i_{n d e x} i(v)=\operatorname{Arg}\left[\operatorname{Max}_{k}\left\{\frac{p_{i}(v) \omega_{k}}{\gamma_{i}(v)} \cdot \chi\left(\frac{p_{i}(v) \omega_{k}}{\gamma_{i}(v)} \leq P M u x\right)\right\}\right]$.

One can note that Eq. (4) considers that mobile $i$ may be the only one to update its power and data rate at a given step; the $\mathrm{SPC}$ is a completely distributed algorithm. Data rate 0 (no transmission) is selected if no transmitted power can be used.

In this paper, we consider the application of the SPC in the environment of WCDMA. The data rates of Section III are used. The SPC is a quality-based power control, i.e., power control update information is based on estimated received quality. In WCDMA System, one information bit is allocated for the update of the transmitted power, at each power control step. Combined power control and rate selection in WCDMA could be achieved in the RRC layer in WCDMA radio protocol architecture.

\section{Simulation results}

This section contains the simulation results. Numerical hypothesis of the simulated system model for the mobile network are now given. The sum of data rates per cell is evaluated in simulation and compared to the same parameter in the Stepwise Removal Algorithm (SRA) of [11].

The SRA applies for a fixed data rate system, in our case $240 \mathrm{~kb} / \mathrm{s}$, which is equivalent to an SIR threshold $\omega_{0}=0.15$ (see Table 1). The SRA is distributed and iterative. The power update formula is: 


$$
p_{i}(v+1)=\frac{p_{i}(v) \omega_{0}}{\gamma_{i}(v)}
$$

Communications are removed until all these remaining achieve their SIR. The data rates (all equal in the case of SRA) of the remaining communications are added in order to have the total throughput.

A fairly simple simulation model is used in order to illustrate the conclusions of this paper. A CDMA system with 25 base stations ( $5 \times 5)$ is considered. The base stations are placed on a rectangular grid. The side of the average square cell is $200 \mathrm{~m}$. Mobiles are uniformly distributed. The link gain between mobile $j$ and base $i$ is modeled as $g_{i j}=c_{s} / d^{4}$ ij where coefficient $c_{s}$ corresponds to obstacles (shadow fading) and $d_{i j}$ is the mobile-to-base distance. Coefficient $c_{s}$ is a zero-mean log-normal random variable whose standard deviation is $\sigma=6 \mathrm{~dB}$. Average values are evaluated by Monte Carlo simulations for 500 independent configurations (mobiles distributions).

Class 3 mobile stations are considered. The maximum transmitted power is $24 \mathrm{dBm}(0.25 \mathrm{Watt})$ and the minimum transmitted power is $-50 \mathrm{dBm}$ as specified in [13]. The receiver thermal noise for the base stations is $-103 \mathrm{dBm}$. A receiver thermal noise is considered in order to have absolute values for transmitted powers computed by the PC algorithms (see Formula (1)). The initial transmitted powers of the algorithms have a random value between the minimum value and $10 \%$ of the (real) value of the maximum transmitted power. A perfect estimation of the received SIR is assumed.

The admission control is not addressed in this work, i.e., an 'admit-all' policy is considered. The two algorithms considered can converge to data rate zero when needed, which is a sort of admission control. The association of the proposed algorithm with an admission control policy is an interesting topic of research. This would, for example, allow the presence of minimum data rate services such as voice services.

We consider a simulation with fixed-position mobiles (snapshot configuration). The convergence of the algorithms is assumed to be fast.enough with regard to the variation of the radio channel to use this condition. After thirty iterations, the values computed by the iterative algorithms used are almost definitive (see below). Given that one iteration corresponds to one time slot $(10 \mathrm{~ms} / 15)$ and that the coherence time of the radio channel is of the order of $100 \mathrm{~ms}$, we can assume the snapshot hypothesis.

According to (3) and (4), data rate zero would lead to a transmitted power equal to zero also, which would not allow a further change of this transmitted power (see update formula (4)). The use of a minimal (non-null) transmitted power, as noted above, eliminates this problem.

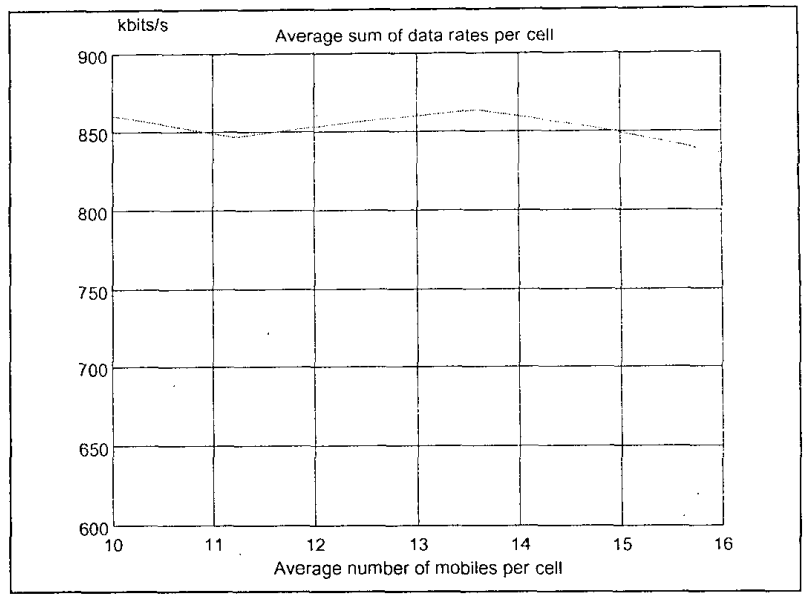

Fig. 2. Sum of the data rates in a cell as a function of the average number of mobiles per cell for the SRA (Stepwise Removal Algorithm)

Figure 2 shows the sum of the data rates in a cell as a function of the average number of mobiles per cell for the SRA. The values of the data rates after 100 iterations are used for the computation of the average sum. At this point, they are sufficiently convergent. Figure 3 shows the same average values for the SPC Algorithm.

We notice in Figure 2 that, in the case of SRA, the average sum of data rates per cell is the same when the number of mobiles per cell changes. This means that (for the high data rates considered) only some mobiles are allowed to transmit while the others are silent. In our case, only 3 or 4 mobiles, in each cell, can transmit at $240 \mathrm{~kb} / \mathrm{s}$.

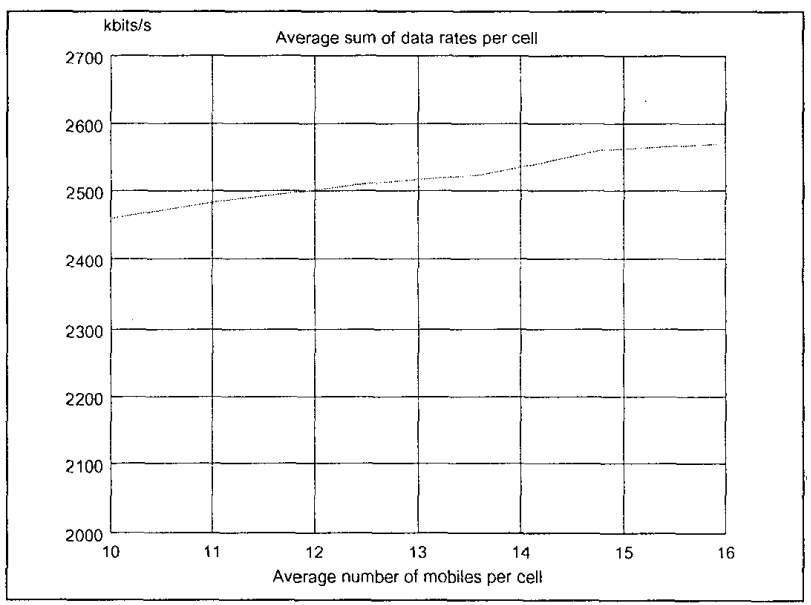

Fig. 3. Sum of the data rates in a cell as a function of the average number of mobiles per cell for the SPC Algorithm: the association of data rate selection and power control leads to a clearly greater sum of data rates per cell. 
Figures 2 and 3 show that the association of data rate selection and power control leads to a clearly greater sum of data rates per cell. In addition, this latter value is no longer fixed when the number of mobiles increases. Having different possible data rates allows greater flexibility. The SPC works in a way such that smaller data rates are used, when needed, in addition to large values which are used whenever it is possible. This allows a higher sum of data rates per cell, a sum that increases when the number of mobiles per cell increases.

The sum of the data rates is plotted for one configuration in Fig. 4 for the SPC Algorithm. The total number of mobiles is 325 (or 13 per cell). Convergence is almost complete after 10 iterations. For a relatively greater number of mobiles (20 or 25 mobiles per cell), the number of iterations for convergence remains smaller than 30 . We verify that the snapshot hypothesis can be assumed with a good approximation.

\section{Conclusions}

In this paper, we have studied the association of power control and data rate selection in a cellular CDMA network. This was done for a specific environment, where different data rates, including data rate zero, can be used, which is a restrictive hypothesis. This is, for example, the case for Internet communications. The computation of the maximal sum of data rates can be useful for any general wireless multimedia CDMA system.

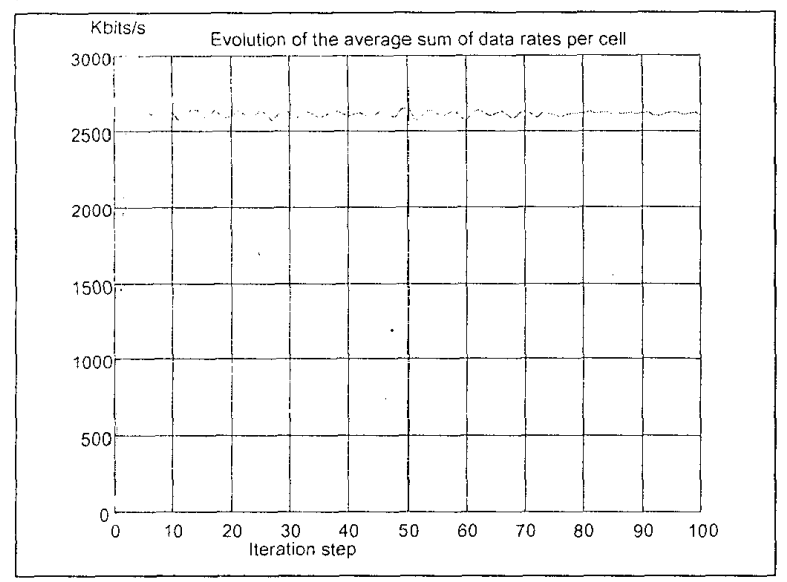

Fig. 4. Sum of the data rates per cell as a function of the number of iterations, plotted for the SPC Algorithm. The total number of mobiles in the simulated area is 325 .

The criterion of optimisation is the instantaneous sum of data rates. We notice that this sum has practically a fixed value for a fixed data rate environment, when the number of demanding (but not necessarily served) mobiles increases. An algorithm with different possible data rates allows greater flexibility. The association of power control and data rate selection works in a way such that smaller data rates are used, when needed, in addition to large values which are used whenever it is possible. With UMTS parameters, the convergence of the iterative algorithm is fast enough to consider that the radio channel is non variable during one data rates values convergence time.

The association of the algorithm studied with an admission control policy is an interesting topic of research. This would, for example, allow the presence of minimum data rate services such as voice transmission. Results of this work can also be used for scheduling policy studies.

Acknowledgement: I would like to thank Ziad El Murr for his help in some of the computer simulations of this work. I also thank Janet Ormrod for her helpful comments.

\section{References:}

[1] A. J. Viterbi, CDMA Principles of spread spectrum techniques. Addison-Wesley, 1995.

[2] H. Holma, A. Toksala, WCDMA for UMTS, Second edition. Wiley, 2002.

[3] 3G TS 25.214 v4.1.0 (2001-06), "Physical layer procedures (FDD) (Release 4)," 3rd Generation Partnership Project; Technical Specification Group Radio Access Networks, Jun 2001.

[4] 3G TS 25.224 v4.0.0 (2001-03), "Physical layer procedures (TDD) (Release 4)," 3rd Generation Partnership Project; Technical Specification Group Radio Access Networks, Mar. 2001.

[5] S.-L. Kim, Z. Rosberg, J. Zander, "Combined power control and transmission rate selection in cellular networks," in Proc. of IEEE Vehicular Technology Conference 1999 Fall, VTC-99F, Sep. 1999.

[6] S. Ramakrishna, J. M. Holtzman, "A Scheme for Throughput Maximization in a Dual-Class CDMA System," IEEE Journal on selected areas in communications, Vol. 16. No. 6, Aug. 1998.

[7] F. Berggren, S.-L. Kim, R. Jäntti, J. Zander, "Joint Power Control and Intracell Scheduling of DS-CDMA Nonreal Time Data," IEEE Journal on selected areas in communications, Vol. 19. No. 10, Oct. 2001.

[8] 3G TS 25.211 v4.5.0 (2002-06), "Physical channels and mapping of transport channels onto physical channels (FDD) (Release 4)," 3rd Generation Partnership Project; Technical Specification Group Radio Access Networks, Mar. 2001.

[9] L. Nuaymi, X. Lagrange, Ph. Godlewski, "A Power Control Algorithm for 3G WCDMA System," in Proc. of European Wireless 2002, Feb 2002.

[10] L. Nuaymi, Contributions for Balanced Power Control Algorithms in Cellular Networks, PhD Thesis, Ecole Nationale Supérieure des Télécommunications (ENST), Paris, 2001.

[11] J. Zander, "Distributed Cochannel Interference Control in cellular radio systems," IEEE Trans. on Vehicular Technology, Vol. 41. No. 3, Aug. 1992.

[12] S. A. Grandhi, R. Vijayan, D. J. Goodman, "Distributed Power Control in Cellular Radio systems," IEEE Trans. on Communications, Vol. 42, Feb/Mar/Apr 1994.

[13] 3G TS 25.101 v4.0.0 (2001-03), "UE Radio Transmission and Reception (FDD) (Release 4)," 3rd Generation Partnership Project; Technical Specification Group Radio Access Networks, Mar. 2001. 